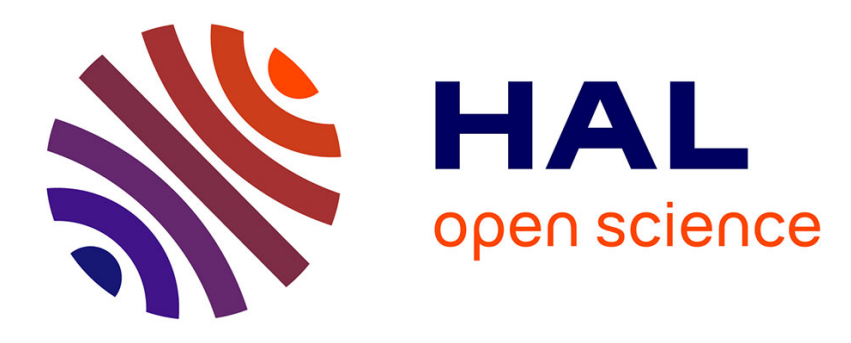

\title{
Norme ISO 9000 et profil du responsable qualité
}

Anne Maurand-Valet

\section{To cite this version:}

Anne Maurand-Valet. Norme ISO 9000 et profil du responsable qualité. Revue Française de Gestion, 2008, 10.3166/RFG.180.61-72 . hal-02537386

\section{HAL Id: hal-02537386 \\ https://hal.umontpellier.fr/hal-02537386}

Submitted on 25 Apr 2020

HAL is a multi-disciplinary open access archive for the deposit and dissemination of scientific research documents, whether they are published or not. The documents may come from teaching and research institutions in France or abroad, or from public or private research centers.
L'archive ouverte pluridisciplinaire HAL, est destinée au dépôt et à la diffusion de documents scientifiques de niveau recherche, publiés ou non, émanant des établissements d'enseignement et de recherche français ou étrangers, des laboratoires publics ou privés. 


\section{NORME ISO 9000 ET PROFIL DU RESPONSABLE QUALITE}

Anne Maurand-Valet, docteur-PRAG, Membre du PRATIC (Programme de Recherche Avignonnais Transdisciplinaire sur l'Internationalisation $d u$ Commerce). Adresse professionnelle: IUT d'Avignon, Site Agroparc, B.P.1207, 84911 AVIGNON Cedex 9. Téléphone : 04908438 10. Adressemèl : anne.maurand-valet@univ-avignon.fr.

Résumé : l'application de la norme ISO 9000 ne peut être réalisée sans la médiation du responsable qualité qui joue un rôle de traducteur de la norme.

Six profils de responsable qualité ont été établis. Ces résultats généraux sont ensuite confrontés au secteur viti-vinicole, spécifique car au carrefour entre artisanat et technologie. On observe alors le cas d'un profil de responsable qualité mixte qui semble particulièrement favorable à la diffusion de la norme.

Mots-clés : norme organisationnelle, responsable qualité, médiation. 


\section{NORME ISO 9000 ET PROFIL DU RESPONSABLE QUALITE}

Résumé : l'application de la norme ISO 9000 ne peut être réalisée sans la médiation du responsable qualité qui joue un rôle de traducteur de la norme.

Six profils ont de responsable qualité été établis. Ces résultats généraux sont ensuite confrontés au secteur viti-vinicole, spécifique car au carrefour entre artisanat et technologie. On observe alors le cas d'un profil de responsable qualité mixte qui semble particulièrement favorable à la diffusion de la norme.

Mots-clés : norme organisationnelle, responsable qualité, médiation.

Face à la multiplication des demandes de certification d'organisations très différentes par leur taille ou leur activité, on peut se demander si cela en vaut la peine et quelles sont les conditions de réussite organisationnelle (Lorino, 1998 ; Laudoyer, 2000).

On constate d'autre part un paradoxe entre d'un côté la volonté des normalisateurs de faciliter le plus possible la mise en place de la norme et le temps que doit y consacrer le responsable qualité en pratique. Ce dernier apparaît comme une personne ressource indispensable à la mise en place de la norme et à son suivi jour après jour.

Il est intéressant de ce fait d'un point de vue pratique de trouver des éléments facilitant le choix du responsable qualité selon le type d'organisation candidate à la certification. 
Une étude sur le rôle de cet acteur dans l'application et la pérennité de la norme ISO 9000 ayant été réalisée sur un ensemble très hétérogène d'organisations, il est apparu intéressant de confronter ces résultats généraux avec ceux observés dans le cadre d'un service technique viti-vinicole qui présente la spécificité de se situer dans un secteur d'activité où l'on conjugue technologie et artisanat. En terme de développement d'instruments liés à la politique qualité cela constitue en effet un contexte très particulier.

Il convient pour pouvoir réaliser cette comparaison :

- de définir ce qu'est une norme socio-organisationnelle et plus particulièrement la norme ISO 9000

- de présenter les résultats généraux obtenus sur l'étude d'ensemble portant sur le rôle du responsable qualité dans la mise en place de la norme ISO 9000

- de situer le cadre dans lequel le service technique vitivinicole exerce son activité

- de mettre en évidence les éléments communs ou divergents entre les deux types de résultats.

- de conclure sur le profil idéal du responsable qualité pour l'entreprise candidate à la certification.

\section{LE RESPONSABLE QUALITE COMME MEDIATEUR} OBLIGATOIRE A LA MISE EN PLACE DE LA NORME ORGANISATIONNELLE ISO 9000

\subsection{LA NORME COMME REPRESENTATION ET OUTIL DE GESTION :}


Grenard (1996) a cherché à caractériser la norme en tant qu'objet et a sélectionné des critères qui permettent de dire si l'on est en présence d'une norme ou non. Ils seraient au nombre de quatre :

- existence d'une spécification technique

- accessibilité au public

- choix collectif préalable

- proposition d'une base d'action pour des problèmes répétitifs

Dans le cas de la norme ISO 9000, le terme de spécification technique utilisé par Grenard ne doit pas être pris au premier degré car il s'agit plutôt pour la norme de fournir une technique au sens de méthode d'organisation et non pas une technique au sens de méthode de production. Dans ce sens-là, la norme ISO 9000 présente bien une spécification technique. En ce qui concerne l'accessibilité au public elle est vérifiée pour la norme ISO 9000 comme pour toutes les normes dont l'objectif final est une diffusion la plus large possible. Le seul obstacle possible est financier (achat de la norme, coût des services des consultants et des audits de certification). La norme ISO 9000 découle enfin d'un choix collectif (Couret, Igalens \& Penan, 1995) et permet de traiter des problèmes répétitifs à travers la gestion documentaire qu'elle organise et la gestion anticipatrice des risques organisationnels dont elle est porteuse.

Une définition de la norme ISO 9000, suffisamment précise pour notre travail sans trop de détails inutiles pour notre réflexion car les approches de la norme sont multiples, pourrait, dans le cadre notre problématique, être celle-ci : La norme est un outil de gestion (David, 1998) cherchant à développer une analyse processuelle de l'organisation afin de mettre en évidence les risques liés à celle-ci qui seront réduits par la formalisation de certaines étapes et l'organisation de la mémoire écrite de l'entreprise. Grâce à cette dernière, un système d'amélioration continue est mis en place sur la base des audits internes. 
Dans l'approche qui est la nôtre nous retiendrons donc comme définition opérationnelle de la norme une représentation de celle-ci à travers trois axes :

- la norme comme organisation de la gestion documentaire

- la norme comme analyse transversale de l'entreprise

- la norme comme réalisation des audits internes en vue de produire une amélioration du système organisationnel.

La norme comme organisation de la gestion documentaire traduit le fait que l'application de la norme ISO 9000 entraîne la mise à plat et la reconsidération de l'établissement, de la circulation, de l'archivage et de la mise à jour de tous les documents au sein de l'organisation. Non seulement cela permet de supprimer les redondances existantes mais aussi de vérifier que les interfaces présentant le plus de risque au sein de l'organisation sont clairement enregistrées sur des documents pour limiter les risques.

La norme comme analyse transversale de l'entreprise met en avant les processus organisationnels. Le texte de la norme insiste sur cette analyse des processus qui se révèle selon les entreprises soit très simple (exemple : concession automobile avec une activité stable au niveau organisationnel et un découpage qui distingue la vente de véhicules neufs, la vente de véhicules d'occasion, la vente de pièces de rechange et le service après vente), soit plus complexe (exemple : aéroport pour lequel les activités sont diverses : mise à la disposition des compagnies aériennes des équipements nécessaires, gestion des parkings passagers, organisation de l'accueil, de l'affichage, promotion de l'aéroport, développement économique de la concession donnée à l'aéroport )

La norme comme réalisation des audits internes en vue de produire une amélioration du système organisationnel traduit le principe d'amélioration continue qui est à la base des systèmes de qualité totale( Krebs \& Mongin, 
2003). Sur eux repose l'auto-évaluation de l'organisation souhaitée par la norme. Réalisés par des acteurs internes à l'organisation, ils doivent permettre de mettre à jour des dysfonctionnements ce qui, une fois ces derniers corrigés, assure une amélioration de l'efficacité de l'organisation dans la poursuite des objectifs qu'elle s'est fixés.

\subsection{La NECESSITE D'UN RESPONSABLE QUALITE POUR LA MISE EN PLACE DE LA NORME ISO 9000 :}

Dans le cadre de la problématique de la médiation du responsable qualité, l'objet de notre recherche était d'illustrer le rôle de ce dernier dans la mise en place et le suivi de la norme ISO 9000 (Bénézech \& Loos-Baroin, 2003).

$\mathrm{Au}$ cours de notre travail d'observation dans 24 organisations différentes (dont 4 à titre exploratoire), à travers des entretiens et en cherchant à croiser deux approches théoriques, d'une part la norme en tant que représentation et d'autre part la norme en tant qu'outil de gestion, il est finalement apparu que l'application de la norme ISO 9000 à une organisation, qu'elle soit privée ou publique, petite ou grande, présente des risques en matière de pérennité que l'activité du responsable qualité cherche à réduire.

Cas étudiés d'entreprises certifiées :

\begin{tabular}{|l|l|}
\hline Effectifs & Secteur d'activité \\
\hline 15 & Formation \\
\hline 49 & Transport \\
\hline 20 & $\begin{array}{l}\text { Fabrication, production et distribution de produits pour la maintenance } \\
\text { industrielle }\end{array}$ \\
\hline 300 & Fabrication, emballage et expédition de laine de verre \\
\hline 50 & $\begin{array}{l}\text { Conception et fabrication de matériel et de logiciel pour l'accueil du } \\
\text { public }\end{array}$ \\
\hline 105 & Production de produits réfractaires \\
\hline 14 & Négoce de produits alimentaires et de liquides \\
\hline 17 & Fabrication et installation de produits préfabriqués en béton \\
\hline 6 & Distribution de matériel médical \\
\hline 49 & Conseil, assistance, production et ingénierie (SSII) \\
\hline 80 & Concessionnaire automobile \\
\hline 900 & Bâtiment, terrassement, travaux souterrains, aménagements urbains \\
\hline
\end{tabular}


Les résultats obtenus peuvent être présentés en trois axes.

D'une part, tous les cas observés ont montré qu'il existait, au delà de l'obtention de la certification, une personne responsable qualité dans les organisations certifiées. Elle pouvait être seule à remplir cette fonction ou être épaulée par une équipe (par exemple la cellule ou le service qualité). Dans certains cas, elle avait comme fonction unique celle de responsable qualité de l'entreprise, dans d'autres elle était polyvalente (responsable de laboratoire, responsable achats, comptable, dessinatrice...)

D'autre part, l'étude de l'action du responsable qualité montre qu'elle concerne autant l'animation du projet de certification (il est, par exemple, l'acteur principal dans la construction de la cartographie) que l'animation au quotidien (prise en charge des revues de direction -l'ordre du jour étant fixé par la norme-, organisation des audits internes et participation personnelle importante à ceux-ci -gestion calendaire des audits afin d'assurer la mise à jour des documents-)

Enfin le responsable qualité joue le rôle de médiateur entre la norme et l'organisation. Il est le lecteur principal si ce n'est unique du texte et présente un statut hybride, à la fois membre interne de l'organisation et externe, en tant que représentant de la norme. Si nécessaire, il traduit les termes spécifiques (Lang, 1992 ; Foucault, 1966) pour en faciliter la diffusion ( " non conformité " devient 
par exemple, " anomalie ", " fiche jaune " ou " fiche d'améliorations possibles "...) et ceci en lien avec son profil personnel.

\subsection{Un ESSAI DE ClaSSification DES PROFILS DES RESPONSAbleS QUALITE :}

On constate sur les 24 cas observés qu'un phénomène de saturation intervient et permet de cerner des profils différents pour les responsables qualité: certains ont un profil plus tourné vers l'écoute de la norme, d'autres vers l'écoute de l'organisation. Cela détermine un comportement de médiation différent. Par exemple, dans une entreprise du secteur secondaire, le responsable qualité généraliste présentant la double casquette de responsable qualité technique et de responsable qualité organisationnelle présente l'analyse des processus demandée par la norme comme évidente à réaliser. Par contre, dans une concession automobile, la personne chargée de la qualité confondra procédure et processus à plusieurs reprises dans son témoignage, termes bien distincts dans la norme.

On observe au final six profils différents qui déterminent des modes de médiation de la norme distincts :

\begin{tabular}{|c|c|c|c|c|c|c|}
\hline & $\begin{array}{l}\text { «Nouveaux } \\
\text { recrutés » }\end{array}$ & $\begin{array}{l}\text { «Anciens } \\
\text { consultants » }\end{array}$ & $\begin{array}{l}\text { « Responsables } \\
\text { qualité } \\
\text { généralistes » }\end{array}$ & $\begin{array}{l}\text { «Idéalistes } \\
\text { communicants » }\end{array}$ & $\begin{array}{l}\text { "Idéalistes } \\
\text { solidaires » }\end{array}$ & $\begin{array}{l}\text { «Famille du } \\
\text { patron » }\end{array}$ \\
\hline $\begin{array}{l}\text { Responsables } \\
\text { qualité } \\
\text { orientés } \\
\text { écoute de } \\
\text { l'organisation }\end{array}$ & & & & $\begin{array}{l}\text { Code } 09 \\
\text { Code } 16\end{array}$ & $\begin{array}{l}\text { Code } 10 \\
\text { Code } 12 \\
\text { Code } 13\end{array}$ & $\begin{array}{l}\text { Code } 11 \\
\text { Code } 06\end{array}$ \\
\hline \begin{tabular}{|l|} 
Responsables \\
qualité \\
orientés \\
écoute de la \\
norme
\end{tabular} & $\begin{array}{l}\text { Code } 05 \\
\text { Code } 14 \\
\text { Code } 15 \\
\text { Code } 19 \\
\text { Code } 22\end{array}$ & $\begin{array}{l}\text { Code 20 } \\
\text { Code 23 }\end{array}$ & $\begin{array}{l}\text { Code } 07 \\
\text { Code } 08 \\
\text { Code } 17 \\
\text { Code } 18 \\
\text { Code } 21 \\
\text { Code } 24\end{array}$ & & & \\
\hline
\end{tabular}


(Les effectifs de chaque catégorie ne peuvent prétendre à aucune représentativité du fait qu'il s'agit d'un échantillon théorique et non représentatif de la population. Ils ne sont là qu'à titre indicatif.)

Nous avons cherché à donner un titre le plus illustré possible et rendant compte du trait caractéristique de chaque catégorie. Ainsi le terme de « nouveaux recrutés » regroupe les cas où le responsable qualité a été embauché dans l'entreprise en vue de prendre en charge le projet de certification ISO 9000. Dans le cas des «anciens consultants » c'est à dire de salariés ayant eu dans le passé une activité de consultant en qualité cette spécificité semble être importante car elle teinte leur discours d'une connotation particulière.

Les catégories des «idéalistes » regroupent des personnes qui semblent posséder des qualités certaines pour jouer le rôle de communicant et de conciliateur dans l'entreprise et qui accordent une grande place aux relations humaines. Nous avons distingué deux sous-groupes : celui des communicants met en avant le profit attendu d'une meilleure communication en terme de transparence, celui des solidaires insiste sur la nécessité dans l'intérêt de l'organisation et du groupe humain qu'elle représente d'obtenir la certification.

La cinquième catégorie de responsables qualité présente la spécificité d'avoir des liens familiaux avec la direction de l'entreprise. Si cela peut sembler normal dans des entreprises petites et assez fréquemment familiales, il est intéressant de le mettre en parallèle avec des commentaires de responsables qualité indiquant que leur fonction est trop souvent perçue comme temporaire car liée à une mission qui aurait une fin dans le temps, celle de la date de certification. Or, ce genre de tâches présente moins de contraintes en terme de gestion du personnel si on l'attribue à un membre de la famille que l'on peut supposer plus souple dans la définition des tâches associées à son poste. Ainsi une fois la mission terminée, on pourra compléter les fonctions du membre de la famille avec les tâches urgentes du moment, ce qui est moins facile avec un salarié ordinaire. D'une certaine manière une responsable qualité intégrée dans le groupe 
«nouveaux recrutés » confirme cette analyse quand elle se présente comme « employée polyvalente »: cette définition de son poste permettrait une fois la mise en place de la norme ISO 9000 réalisée de modifier son affectation.(si l'on reprend le raisonnement de son employeur).

Enfin, la sixième catégorie regroupe les responsables qualité qui, par leur activité première, étaient déjà concernés par la qualité, mais uniquement dans sa dimension qualité du produit (responsable laboratoire, technicien, pharmacienne dans l'industrie, docteur en chimie, ingénieur)

Armés de ces résultats, il paraît intéressant de les comparer au cas très spécifique du secteur viti-vinicole pour voir l'impact de cette activité qui est marquée à la fois par une dimension technologique et artisanale. On peut s'interroger en effet sur le choix du profil du responsable qualité dans ce contexte particulier.

\section{APPLICATION DES RESULTATS A UN SERVICE TECHNIQUE VITI-VINICOLE :}

Le cas de ce service technique viti-vinicole a été étudié à travers l'analyse d'entretiens réalisés auprès du responsable qualité et à travers une étude documentaire des textes de présentation de cette organisation, des normes qui y sont appliquées et de son manuel qualité.

\subsection{LE CONTEXTE :}

Le service technique auquel nous nous sommes intéressés appartient à un organisme para-public de statut privé mais fonctionnant sous la tutelle de 
ministères et contrôlé par l'Etat. Il bénéficie entre autres d'une délégation de la Direction Générale de la Concurrence, de la Consommation et de la Répression des Fraudes pour le suivi aval de la qualité.

Il a été créé à la demande des professionnels de la viticulture et du négoce par la loi du 28 novembre 1955. En tant qu'organisme interprofessionnel, il a pour mission de procéder à toutes les études et de centraliser toutes les statistiques permettant la connaissance de l'offre et de la demande ainsi que l'adaptation et la régularisation de l'offre. Il met en œuvre, sous le contrôle de l'Etat, les règles de mise en marché et de conditions de paiement. Il réalise la promotion de toutes les mesures d'ordre scientifique et technique susceptibles d'améliorer la qualité des produits et il organise en France et à l'étranger la promotion des vins A.O.C. L'organisme interprofessionnel auquel appartient notre service technique est construit sur trois principes fondamentaux :

- la représentativité des familles professionnelles

- l'équilibre paritaire entre les familles professionnelles du négoce et de la viticulture

- l'unanimité pour toute décision majeure prise par l'Interprofession.

L'effectif global de l'organisme interprofessionnel est actuellement de 50 personnes, 22 travaillant dans le service technique que nous avons étudié. Au début l'activité était essentiellement tournée autour de l'activité de laboratoire ( analyses œnologiques, micro-biologiques, fines pour le suivi et le contrôle de la qualité des vins). Puis l'accent a été mis sur la qualité et le service technique a développé une activité de conseil et de formation ainsi que le suivi aval de la qualité en lien avec la DGCCRF. Parallèlement les missions de communication et de promotion des vins se sont aussi développées.

Pour faire face à ces nouvelles activités, le service technique a été amené à demander certaines accréditations ou certifications. 
Une première étape a été franchie avec l'obtention de l'accréditation pour la norme ISO 17025. Elle regroupe les prescriptions générales concernant la compétence des laboratoires d'étalonnages et d'essais et est rendue obligatoire par l'Etat pour obtenir une délégation de la DGCCRF.

La seconde étape a été abordée quand le consultant a convaincu l'encadrement de l'intérêt d'intégrer tout le service technique dans le périmètre de certification du système de management. Fin 2002, il est donc décidé de faire certifier ISO 9000 tout le service technique ce qui est chose faite 12 mois plus tard. D'après la responsable qualité, le principal intérêt de cette démarche a été d'obliger l'organisation à repenser ses métiers et à les redéfinir. Entre autres, le problème qui se pose pour ce type d'organisations multi-partenaires est celui de la définition de ses clients. Posées au carrefour de nombreux réseaux (les professionnels de la production et du négoce, l'Etat, les clients, les revendeurs), les multiples forces qui s'exercent risquent de faire perdre aux organisations leur homogénéité et leur cohérence. La mise à plat de leurs missions dans le manuel qualité, l'inscription de leurs clients et le découpage de leur activité à travers l'analyse en terme de processus demandée par la norme ISO 9000 est l'occasion de développer une communication interne sur les objectifs premiers de ces organisations. Cela permet de rapprocher les représentations qu'en ont les acteurs et donc de resserrer la cohérence du groupe.

\subsection{LE PROFIL DU RESPONSABLE QUALITE :}

Par rapport aux six profils types relevés lors de l'étude générale précédente, la responsable qualité du service technique étudié présente un cas hybride. En effet, elle apparaît à la fois comme une responsable qualité généraliste puisqu'elle a débuté dans le laboratoire au niveau de la qualité du produit, ici le vin, mais elle est aussi une idéaliste communicante car elle axe 
son discours sur le bénéfice que l'on peut retirer d'une plus grande transparence dans l'organisation elle-même et dans les relations entre l'organisation et ses partenaires. Elle associe ainsi la parfaite maîtrise de la qualité du produit, une bonne connaissance de l'organisation et une perception valorisante de l'application de la norme. En effet, elle a au départ une formation en œnologie qui l'a amenée à être recrutée 26 ans auparavant pour travailler dans le laboratoire d'analyse qui représentait alors la quasi-totalité de l'activité de l'organisation interprofessionnelle. L'effectif était de 4 dans le service technique, pour 22 personnes actuellement (50 pour la totalité de l'organisation interprofessionnelle). La responsable qualité a débuté dans la fonction qualité en 1989. En effet, à cette époque-là, l'Union Française des Enologues, regroupement de professionnels bien structurée, avait décidé d'engager une démarche collective en qualité afin de mettre à la disposition de tous ces membres une démarche et un manuel qualité types (les laboratoires œnologiques étant très petits il semblait obligatoire de mutualiser les moyens). Ce projet a fait intervenir des consultants et les responsables des laboratoires. L'accréditation à la norme ISO 17025 pour le service technique a ainsi été obtenue en 1993. La responsable qualité s'est alors recyclée dans la formation qualité pour aider les entreprises viti-vinicoles à décrocher la certification ISO 9000 tant il semblait à ce moment-là que le certificat était le passage obligé pour assurer la pérennité de toutes les entreprises (depuis la profession semble d'accorder sur le fait qu'il ne s'impose pas pour les petites exploitations qui n'ont besoin que des certificats garantissant la qualité produit. L'assurance qualité en management se limite ainsi aux organisations plus importantes). Néanmoins, durant 4 ans, notre responsable qualité s'est associée avec des consultants pour proposer, conformément aux missions de l'organisation interprofessionnelle, une aide dans la démarche ISO 9000 aux entreprises viti-vinicoles.

En 1995, elle a débuté par ailleurs une activité de communication, pôle négligé jusque là par l'organisation et a contribué à la création du service de suivi aval 
de la qualité (délégation donnée par la DGCCRF). Elle en a été désignée comme responsable.

$\mathrm{Au}$ total, il ressort de son cursus une capacité à s'adapter aux besoins qui découlent de l'évolution de l'organisation. Elle est la personne polyvalente à $100 \%$, maitrisant à la fois tout l'aspect technique du produit et connaissant sur le bout des doigts le fonctionnement de l'organisation. Quand elle décrit le développement et l'évolution de cette dernière, elle le fait par ailleurs avec une fluidité dans son témoignage qui montre la maîtrise qu'elle possède de l'historique et des facteurs qui ont influencé la vie de l'organisation.

Du fait de sa nature hybride, à la fois « responsable qualité généraliste » et «idéaliste communicante», elle se trouve être à l'écoute simultanément de l'organisation et de la norme. Elle présente ainsi un profil équilibré ce qui présente un facteur favorable à la mise en place de la norme ISO 9000 (qu'elle a réussi puisque la certification a été obtenue) et à la pérennisation de l'application. Cela apparaît entre autres à travers l'accent qu'elle met sur la nécessité de faire vivre le système qualité de management et la présentation qu'elle fait de la revue de direction comme levier efficace dans la demande de moyens vis à vis du bureau de l'organisation professionnelle. Elle montre comment ce rendez-vous annuel qui permet un bilan des actions passées et la fixation des objectifs pour l'avenir fournit l'occasion au service technique de défendre ses positions stratégiques grâce aux données chiffrées et argumentées établies grâce aux enregistrements demandés par la norme ISO 9000. La structuration de l'activité de management qui résulte de l'application de la norme est pour la responsable qualité un avantage important face au bureau et à l'assemblée générale d'élus, ainsi qu'à la multiplicité des partenaires. Ceci contribue à donner une légitimité à l'application de la norme aux yeux des personnes travaillant dans le service technique.

La responsable qualité indique par ailleurs que grâce à la mise en place de la norme ISO 9000 le service technique a pu survivre à un déménagement, un 
regroupement de laboratoires et trois changements de direction en huit ans. Le système qualité a été un rempart tant du point de vue politique qu'organisationnel.

\subsection{LES BENEFICES DE LA MIXITE DU PROFIL DU RESPONSABLE QUALITE :}

Dans le cas que nous venons de décrire, le responsable qualité présente un profil à la fois de «responsable qualité généraliste » et d' «idéaliste communicant » :

- c'est une ancienne technicienne du laboratoire, ce qui lui donne toutes les compétences concernant la qualité du produit

- elle transmet à travers son discours une vision moderniste de la norme fondée sur la transparence et l'efficacité qui seraient apportées par celleci.

Elle se trouve de ce fait simultanément à l'écoute de l'organisation, à travers son cœur de métier, le vin, et à l'écoute de la norme, puisqu'elle en véhicule l'image première utilisée pour la promotion de celle-ci, la modernité. Ainsi elle a pu joué pleinement son rôle dans les deux étapes de l'application de la norme :

- d'une part au moment de la demande de certification

- d'autre part dans la gestion au quotidien de l'application.

En effet, l'obtention de la certification nécessite la constitution d'un dossier et la réussite à l'audit de certification. Le responsable qualité joue à ce niveau-là un rôle important car le projet doit être soutenu si l'on souhaite le voir aboutir. L'analyse transversale de l'organisation, préalable au traçage de la cartographie des processus demandée par la certification, et l'analyse en terme de clients suscitent une mise à plat de l'image que les acteurs ont de leur organisation. La responsable qualité souligne la nécessité d'amener les personnes à repenser les métiers et à les redéfinir, mais plus encore, (« le plus intéressant pour nous » dit- 
elle), à déterminer qui sont les vrais clients de l'organisation. Il faut en effet identifier clairement ceux-ci pour analyser ensuite leur satisfaction comme l'impose la norme ISO 9000. Or dans une organisation multi-partenaires comme le service technique étudié, la réflexion sur le concept de clients est particulièrement difficile à réaliser (on peut opposer ce cas à celui des concessions automobiles que nous avons eu l'occasion d'aborder dans l'étude générale. Elles présentent la particularité d'avoir des activités stables dans le temps et des clients facilement identifiables. L'analyse des processus en est simplifiée. Elle ne modifie pas la représentation que les acteurs ont de leur organisation). De plus, pour établir la cartographie des processus, la responsable qualité a joué un rôle d'intermédiaire entre l'organisation et le consultant sollicité car ce dernier était étranger aux métiers viti-vinicoles (ce qui semble par ailleurs positif à notre responsable qualité qui note qu'il n'avait pas d'a-priori et qu'il s'est contenté de poser les “bonnes questions"). Elle a pu joué ce rôle grâce aux connaissances techniques qu'elle détenait.

Mais si le responsable qualité joue un rôle essentiel dans l'obtention de la certification, il est aussi l'acteur primordial dans la gestion au quotidien de l'application. En effet, le risque est celui d'une perte de sens de l'application, d'une occultation par d'autres priorités et d'un échec de l'audit de renouvellement. Elle peut aussi être phagocytée par le fonctionnement routinier de l'organisation, perdre toute sa spécificité et disparaître par effacement progressif de ses principes logiques essentiels dans l'esprit des acteurs.

Dans le service technique étudié, la responsable qualité utilise l'application comme un outil de communication vis à vis de la direction générale de l'organisation interprofessionnelle. En effet, la norme prévoit des revues de direction au moins une fois par an qui sont l'occasion de dresser des bilans et de fixer des objectifs pour l'avenir. Ces revues de direction sont alimentées en données grâce aux enregistrements demandés par la norme qui servent à assurer 
une traçabilité des activités. En structurant de manière cohérente la collecte de ces données par rapport à la logique des revues de direction, la responsable qualité peut ainsi, en présence du directeur général de l'organisation interprofessionnelle, défendre les intérêts du service technique et prouver la nécessité des moyens demandés, le directeur général étant lui-même moins bien informé de la situation.

En définitive, on constate que, et les compétences techniques, et les compétences dans la communication de la vision moderniste de la norme, tendent à conférer à la responsable qualité une grande efficacité dans la mise en place et dans la pérennisation de l'application.

Nous avions observé l'existence de six profils distincts dans une étude générale préalable : «nouveaux recrutés », « anciens consultants », « responsables qualité généralistes », «idéalistes communicants », « idéalistes solidaires » et «famille $\mathrm{du}$ patron ». L'approche d'un terrain très particulier, celui viti-vinicole, au carrefour entre artisanat et technologie, nous a permis de voir apparaître un profil mixte, combinaison des deux profils « responsable qualité généraliste » et « idéaliste communicant». Il permet de conjuguer écoute de l'organisation et écoute de la norme. Cette mixité semble profitable en terme d'efficacité organisationnelle. D'autres combinaisons seraient possibles comme celle de « responsable qualité généraliste » et d'« idéaliste solidaire ».

Par contre il semble difficile de croiser «nouveau recruté » ou «ancien consultant » avec «idéaliste solidaire » car ce dernier profil va le plus souvent de pair avec une ancienneté importante dans l'organisation.

Mais à vouloir un responsable qualité présentant toutes les qualités à la fois, on peut aussi aboutir à la recherche d'un « mouton à cinq pattes ».

Pour éviter cette situation, le contexte organisationnel peut contribuer à sélectionner le responsable qualité : 
- dans le cas d'a-priori organisationnels défavorables à la mise en place de la norme, les responsables qualité seront peut-être d'autant plus facilement acceptés qu'ils auront un profil plus tourné vers l'écoute de l'organisation (« idéalistes communicants », «idéalistes solidaires » et «famille du patron »)

- si, par contre, les a-priori sont favorables, un responsable qualité tourné vers l'écoute de la norme pourrait obtenir plus rapidement la certification du fait de ses connaissances immédiatement mobilisables du modèle à mettre en place.

\section{Bibliographie :}

BENEZECH D. \& LOOS-BAROIN J., « Le processus de certification ISO 9000 comme outil d'apprentissage organisationnel », Revue Sciences de Gestion , n³6, printemps 2003, pp.1143.

BOLTANSKI L \& THEVENOT L., De la justification, Les économies de la grandeur, Edition Gallimard, 1991.

CHAMBON R., Le monde comme perception de la réalité Librairie philosophique J.Vrin, 1963.

CORBIN J \& STRAUSS A., Basics of Qualitative Research,Techniques and Procedures for Developing Grounded Theory, Edition Sage Publications, second edition, 1998.

COURET A., IGALENS J. \& PENAN H., La certification, Collection Que Sais-je ?, PUF, 1995. 
COURPASSON D., «Les normalisations managériales entre l'individu et le modèle professionnel », Revue d'Economie Industrielle, n75, 1er trimestre 1996, pp.239-256.

DAVID A., «Outils de gestion et dynamique de changement », Revue Française de Gestion, septembre-octobre, pp.44-58, 1998.

FOUCAULT M., Les mots et les choses, une archéologie des sciences humaines, NRF, Edition Gallimard, 1966.

GOMEZ P.Y., «Normalisation et gestion de la firme : une approche conventionnaliste », Revue d'Economie Industrielle, nº75, 1er trimestre 1996, pp.113-131.

GRENARD A., «Normalisation, certification: quelques éléments de définition », Revue d'Economie Industrielle, $\mathrm{n}^{\circ} 75$, 1er trimestre 1996, pp.45-60.

KREBS G. \& MONGIN Y., Les nouvelles pratiques de l'audit interne, Edition AFNOR, 2003.

LANG P., «Parcours linguistique de discours spécialisés », Actes du colloque en Sorbonne, Paris, 1992, Sciences pour la communication.

LAUDOYER G., La certification ISO 9000. Un moteur pour la qualité, Les Editions d'Organisation, 2000.

LORINO P., Méthodes et pratiques de la performance, Editions d'Organisation, 1998. 
